\title{
Field Shape Measurements of Prototype Main Injector Dipole Endpacks
}

\author{
Henry D. Glass, Bruce C. Brown and David J. Harding \\ Fermi National Accelerator Laboratory \\ P.O. Box 500, Batavia, Illinois 60510
}

December 1992 


\section{Disclaimer}

This report was prepared as an account of work sponsored by an agency of the United States Government. Neither the United States Government nor any agency thereof, nor any of their employees, makes any warranty, express or implied, or assumes any legal liability or responsibility for the accuracy, completeness, or usefulness of any information, apparatus, product, or process disclosed, or represents that its use would not infringe privately owned rights. Reference herein to any specific commercial product, process, or service by trade name, trademark, manufacturer, or otherwise, does not necessarily constitute or imply its endorsement, recommendation, or favoring by the United States Government or any agency thereof. The views and opinions of authors expressed herein do not necessarily state or reflect those of the United States Government or any agency thereof. 


\title{
Field Shape Measurements of Prototype Main Injector Dipole Endpacks
}

\author{
Henry D. Glass Bruce C. Brown \\ David J. Harding \\ Fermi National Accelerator Laboratory * \\ P.O. Box 500 \\ Batavia, Mlinois 60510 \\ January 6, 1993
}

\begin{abstract}
Measurements of the transverse dependence of the flux on the symmetry plane were obtained on a series of endpacks mounted on a Main Injector prototype dipole. From these flux measurements, we determined the endfield shape, expressed in terms of normal harmonics, up to 14-pole. We describe the measurement and analysis procedure, and present the results for all endpacks that were tested. The final endpack (number 10) has a sextupole, normalized to the body, of $+0.167 \pm .072$ units, and the relative field shape deviates by $<1.2$ units relative to the on-axis field strength over the range $|x|<2.0^{\prime \prime}$. These measurements indicate that Endpack 10 meets the requirements for the Main Injector dipole.
\end{abstract}

\section{Introduction}

We report measurements of field shapes for a number of endpacks which were mounted on the non-lead end of magnet IDM002, the 2nd prototype dipole for the Fermilab Main Injector. Normal harmonics were estimated from the field shapes by a least squares fit.

Data were acquired using the $80^{\prime \prime}$ Flatcoil fiux measuring probe. Some details regarding the probe and the method of data acquisition can be found

\footnotetext{
-Operated by the Univeraities Research Association under contract with the U. S. Department of Energy
} 
in a companion report[1]. For this series of measurements, the probe was utilized in the scan mode, in which the magnet current is rept constant and the probe scans horizontally across the magnet aperture, measuring

$$
\Delta \Phi(x, i)=\Phi(x, i)-\Phi(0, i)
$$

The absolute flux at $x=0$ is measured using the probe in the baseline mode (see ref. [1]). By positioning one end of the probe a depth $z_{0}$ inside the magnet (the other end being outside the magnet), we measured the field integral as a function of depth and transverse position:

$$
J\left(x, z_{0}\right)=\int_{-\infty}^{z_{0}} B(x, z) d z
$$

The sections below describe how the endfield harmonics are estimated from measurements of $J\left(x, z_{0}\right)$.

\section{Measurement Procedures}

At each selected current $(500,1500,7000$, and $9500 \mathrm{~A}$ were the nominal currents) we measured $J(x, z)$ over the longitudinal range $0<z<20^{\prime \prime}$ in $2^{n}$ steps. Measurements were controlled using the MTF CAMAC/VAX measurement system running the FLATCOIL program. At each $z$ position we scanned transversely from $-2.5^{\prime \prime}<x<+2.5^{\prime \prime}$ in $0.100^{\prime \prime}$ steps. Four scans were made at each $z$, and for each $x$ position we recorded the average of the four scans and the standard deviation in the data file. For the first several endpacks we oriented the probe normal to the face of the magnet. This introduced a small error due to the magnet curvature which we corrected to some extent in the off-line analysis. Beginning with Endpack 7, we inserted the probe at an angle of $0.6^{\circ}$ with respect to the lamination face. This angle coincides with the beam direction.

Reference [1] should be consulted for brief descriptions of each endpack. Because the field shape measurements required the acquisition of considerably more data than for the effective length portion of the measurements, we decided not to measure the field shape of every endpack, but only those for which we expected a substantial change with respect to the previous endpack. Endpack 1 was measured with a technique different from the one reported here. We expect it's shape to be very similar to Endpack 2. The endpacks for which we report harmonics measurements in this report include numbers $2,3,5,7,8,9$, and 10 . 


\section{Data Analysis}

\subsection{Body field / End field separation}

The aim of the first part of the data analysis is to separate the component of the flux that is attributable to the end field from the body field. For probe positions $z>z_{\min }$, where $z_{\min }$ is the location inside the magnet beyond which end effects are unimportant, we can make a linear approximation to the field integral as a function of $z$ :

$$
J(x, z)=\alpha(x)+\beta(x) z
$$

The function $\beta(x)$ can be identified as the body field shape, $B(x)$. We can identify $\alpha(x)$ as the effective end field integral:

$$
\alpha(x)=\int_{-\infty}^{\text {end }} B(x, z) d z
$$

That is, it is the field integral over some region containing the end of the magnet, with the body contribution subtracted. Note that we do not specify precisely what the upper limit on this integral is, nor do we need to, as long as the probe integrates over a region at least as large as any region in which end effects are important. This is equivalent to choosing $z_{\min }$ large enough so that $B\left(z_{\min }\right)$ contains only body field. For this analysis we chose $z_{\min }=10^{\prime \prime}$.

Prior to performing fits to $J(x, z)$ at each $x$ position, we corrected the data for the misalignment of the probe with respect to the magnet axis. This is done by adding an offset to the $x$ position which increases linearly in $z$ :

$$
x(z)=x_{0}+x_{\text {offeet }} z
$$

The field integrals $J(x, z)$ are then interpolated at the nominal $x$ positions $(-2.0,-1.9,-1.8 \ldots 0.0 \ldots 1.9,2.0)$ using a quadratic interpolation technique. The value of $x_{\text {offict }}$ was typically $0.004^{n}$. Note that this interpolation was no longer necessary beginning with the Endpack 7 data.

The error estimated for each $J(x, z)$ was determined from the measured standard deviation in the flux and an estimate of the $z$ positioning error. The $z$ position error was typically $0.005^{n}$ (see discussion in [1]). For each $x$ position we wrote to a data file the fits to the coefficients $\alpha$ and $\beta$ and the estimated errors in the fits, and an estimate of the goodness of fit $\left(\chi_{\nu}^{2}\right)$. 


\subsection{Endfield shapes}

Figures 1 through 7 show the relative shapes of the endfields. These shapes are the endfield integrals of Equation 4 normalized by the quantity $B_{0} L$ and multiplied by $10^{4}$. The endfield shapes are observed to be approximately independent of current. The distinct two-hump shape seen in many of the endfields can be parameterized by a large positive sextupole combined with a weaker negative decapole. The magnitude of the sextupole is correlated with the size of the noses on the endpacks. Endpack 3, which has no noses or shims, is the only one observed to have a natural negative sextupole, and no humps in its shape. Some degree of saturation is observed at higher currents beginning with Endpack 5, resulting in a gradual depression of the size of the humps as current is increased.

Figure 8 shows a superimposed view of all Endpacks at 1500 A. Endpack 10 is seen to have the most desirable shape, in that its deviation from zero is smaller than any of the others. 


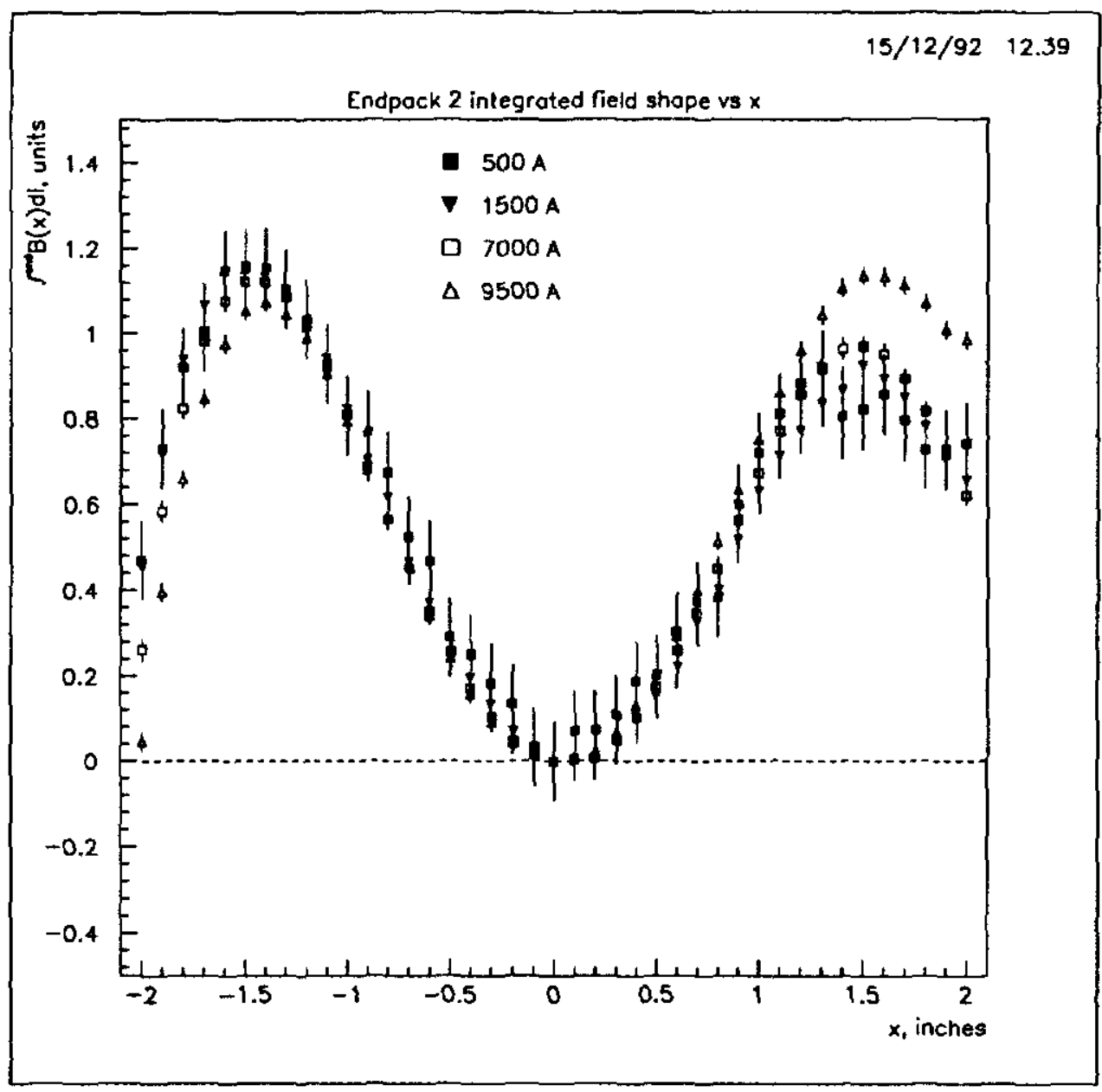

Figure 1: Integrated field shapes for Endpack 2. The data plotted here and in subsequent figures are $10^{4} \times \alpha(x) /\left(B_{0} L\right)$ vs $x$ 


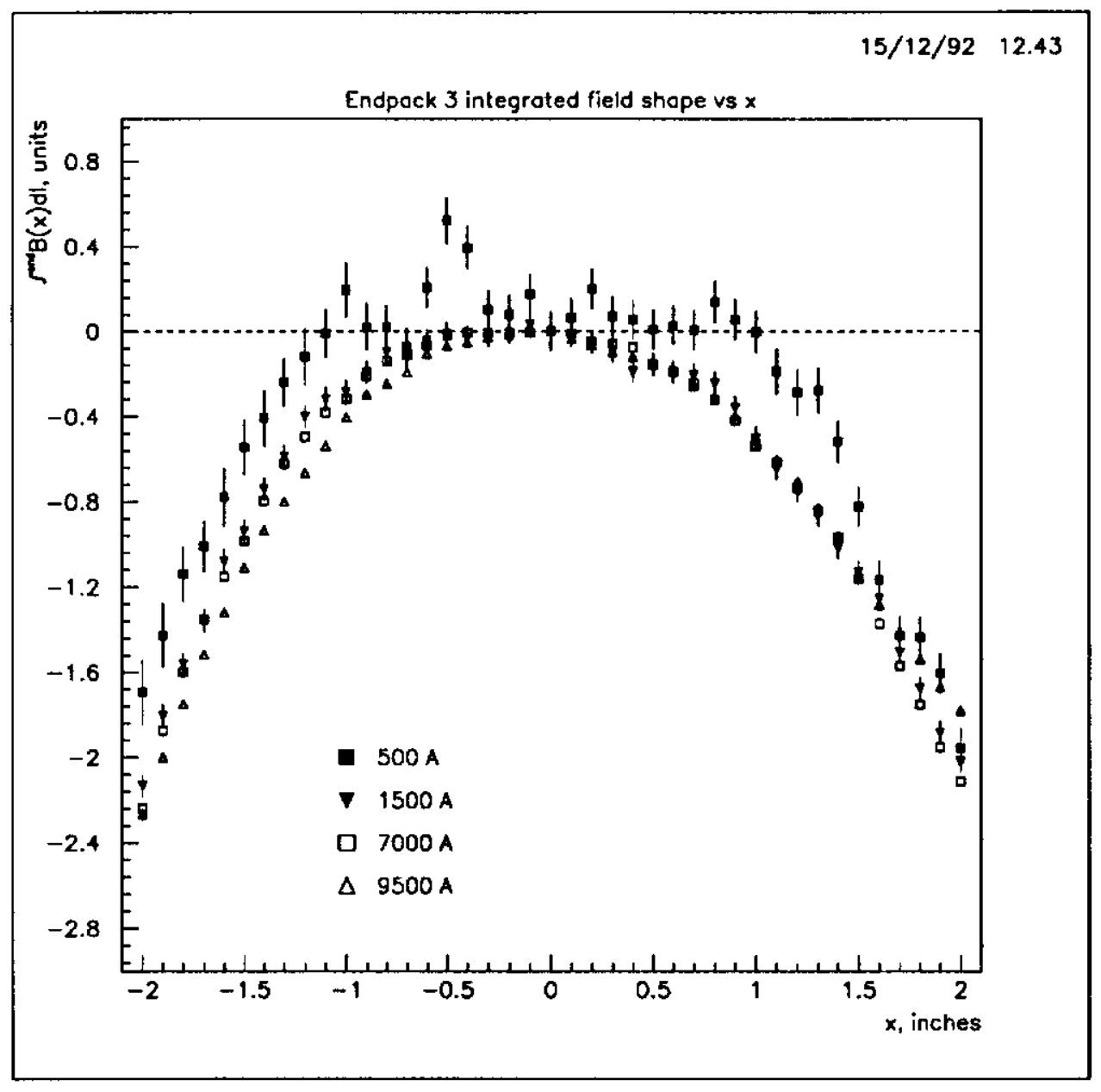

Figure 2: Integrated field shapes for Endpack 3. 


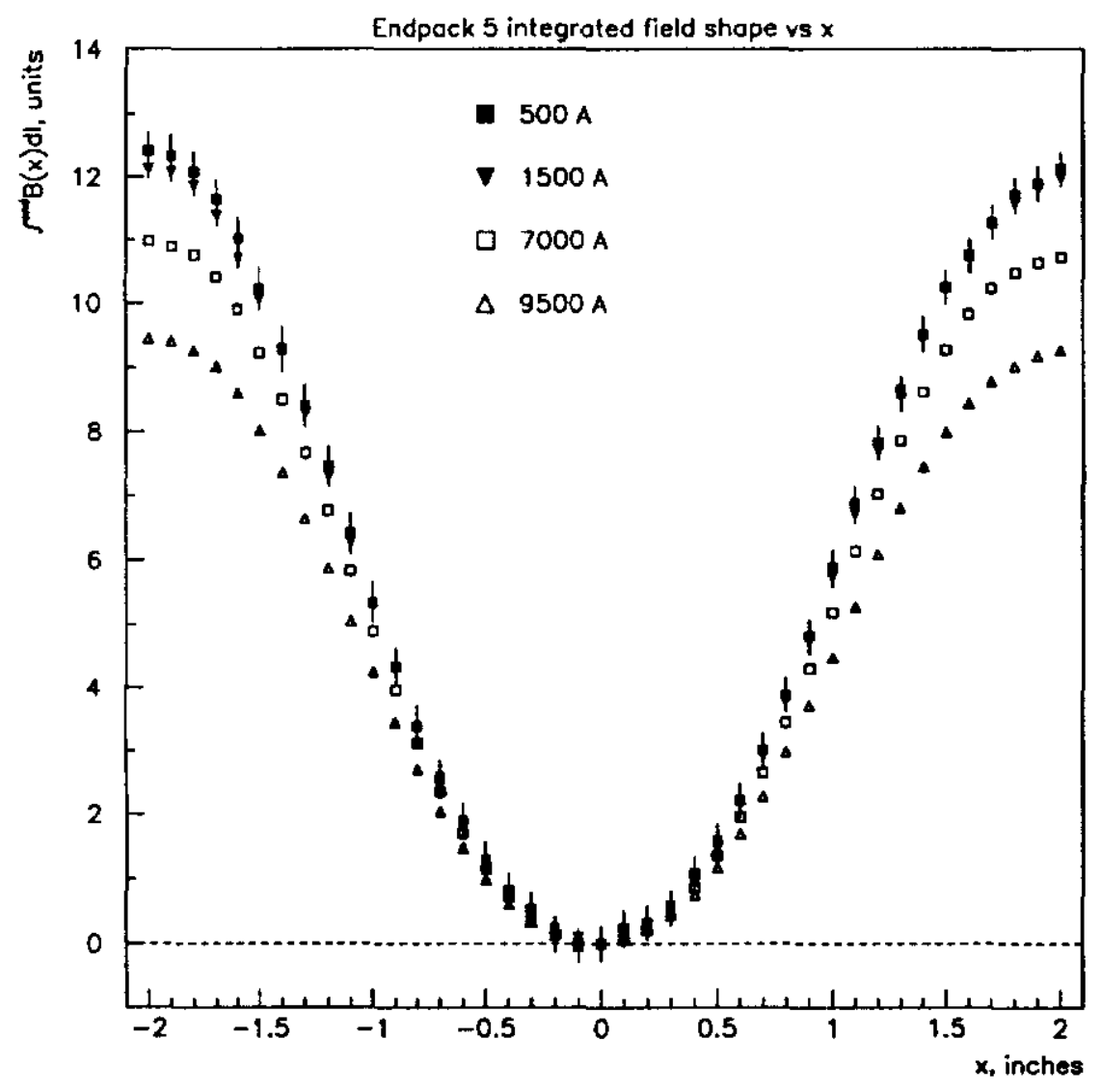

Figure 3: Integrated field shapes for Endpack 5. 


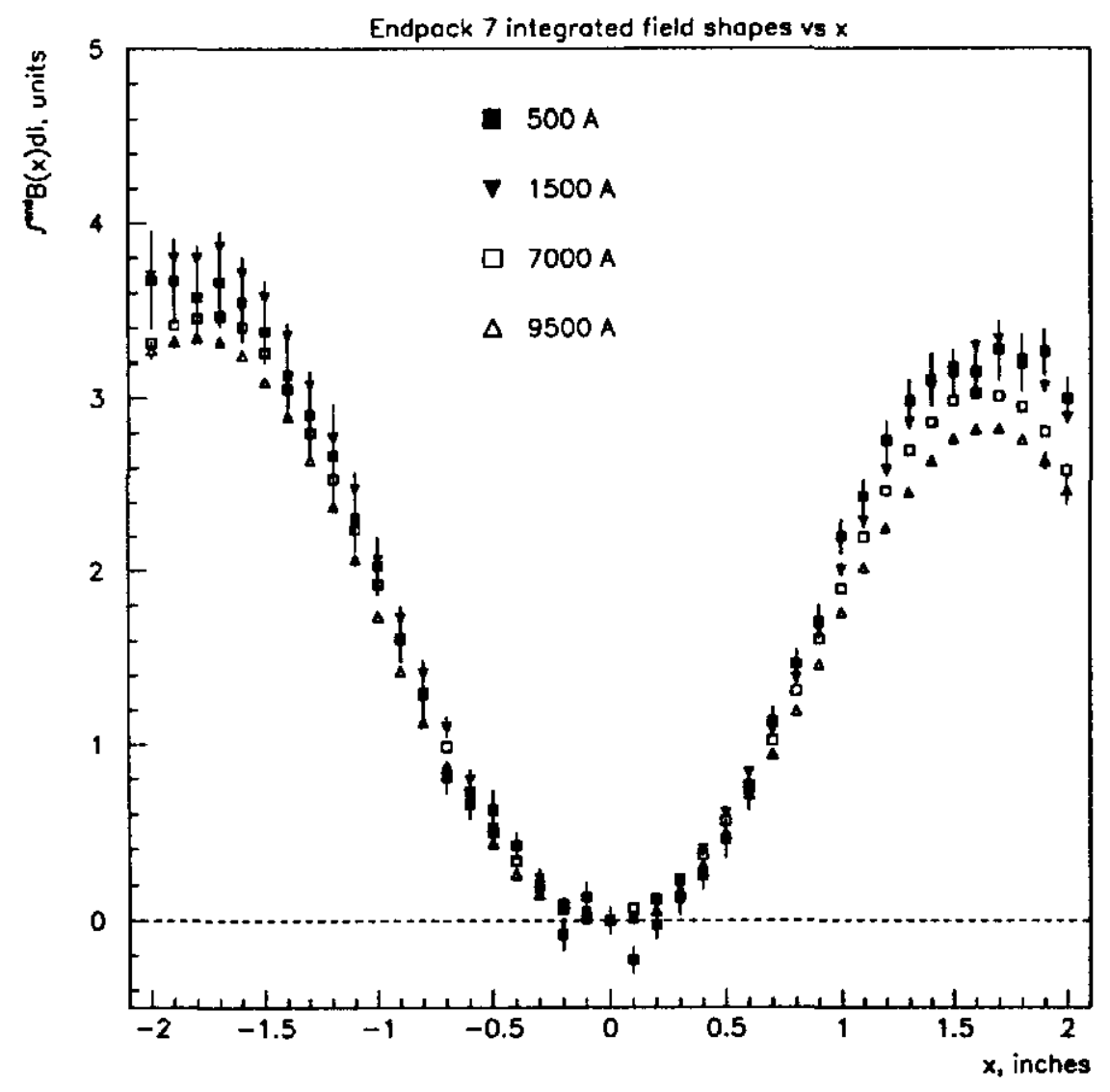

Figure 4: Integrated field shapes for Endpack 7. 


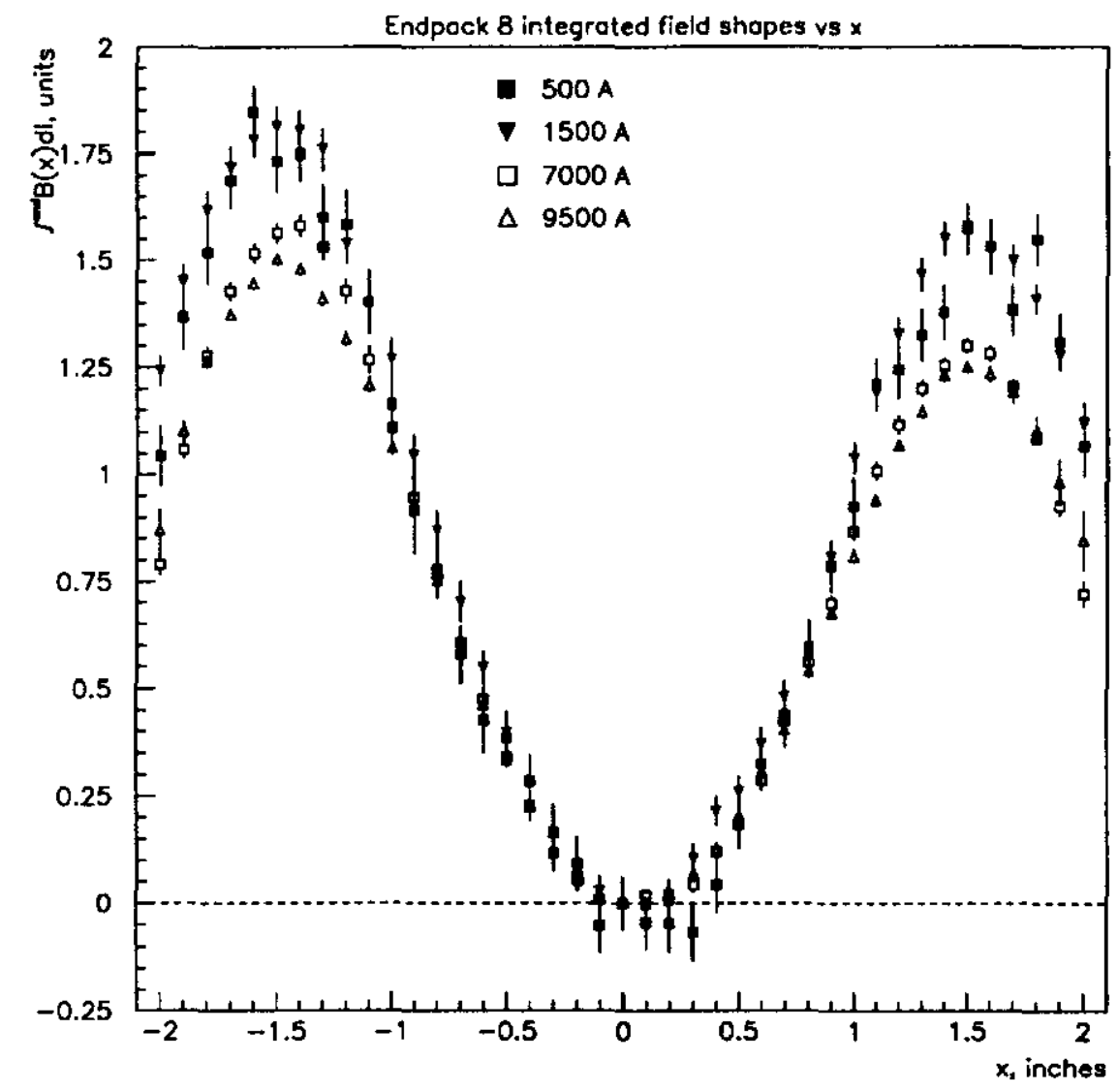

Figure 5: Integrated field shapes for Endpack 8. 


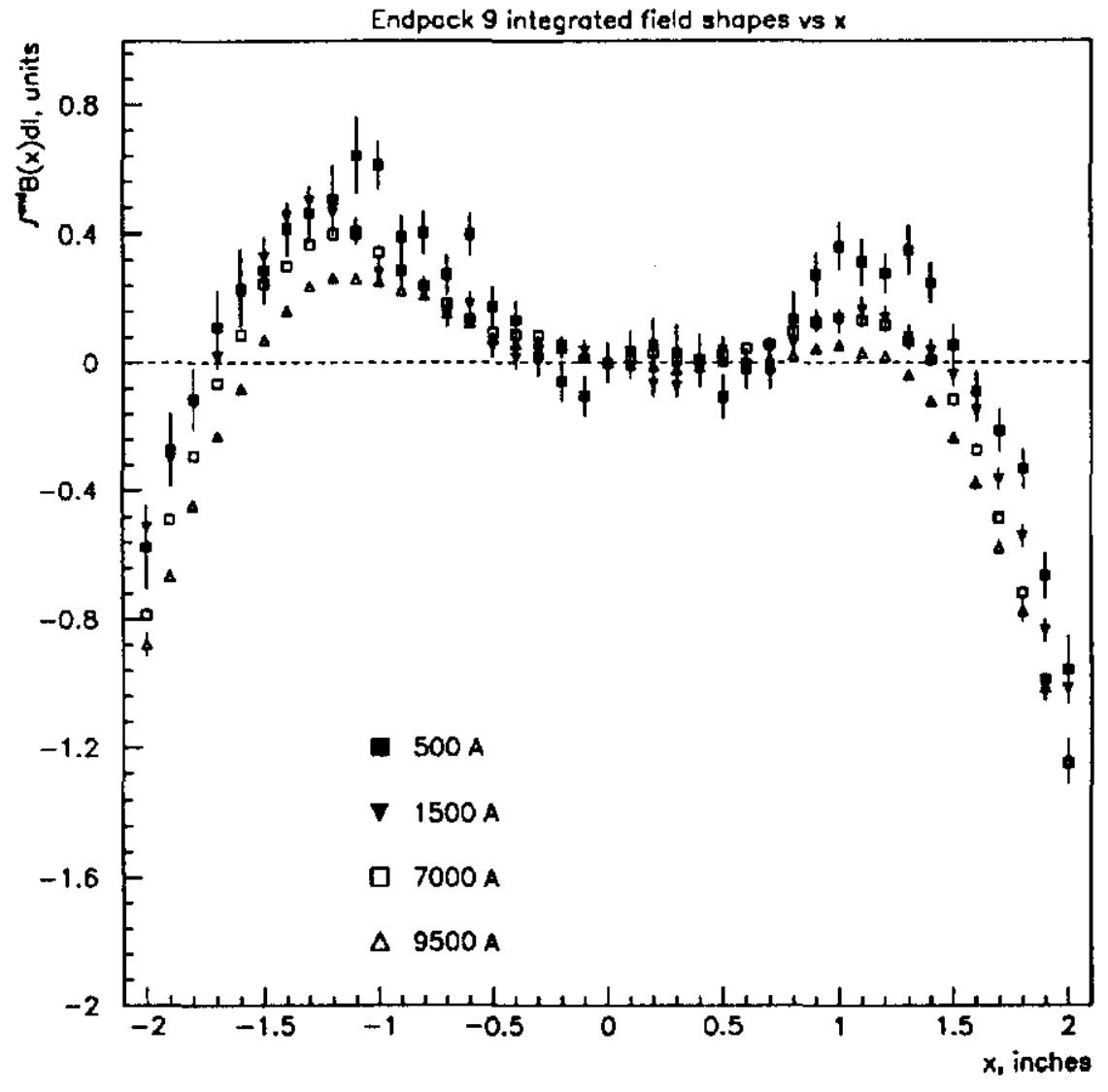

Figure 6: Integrated field shapes for Endpack 9. 


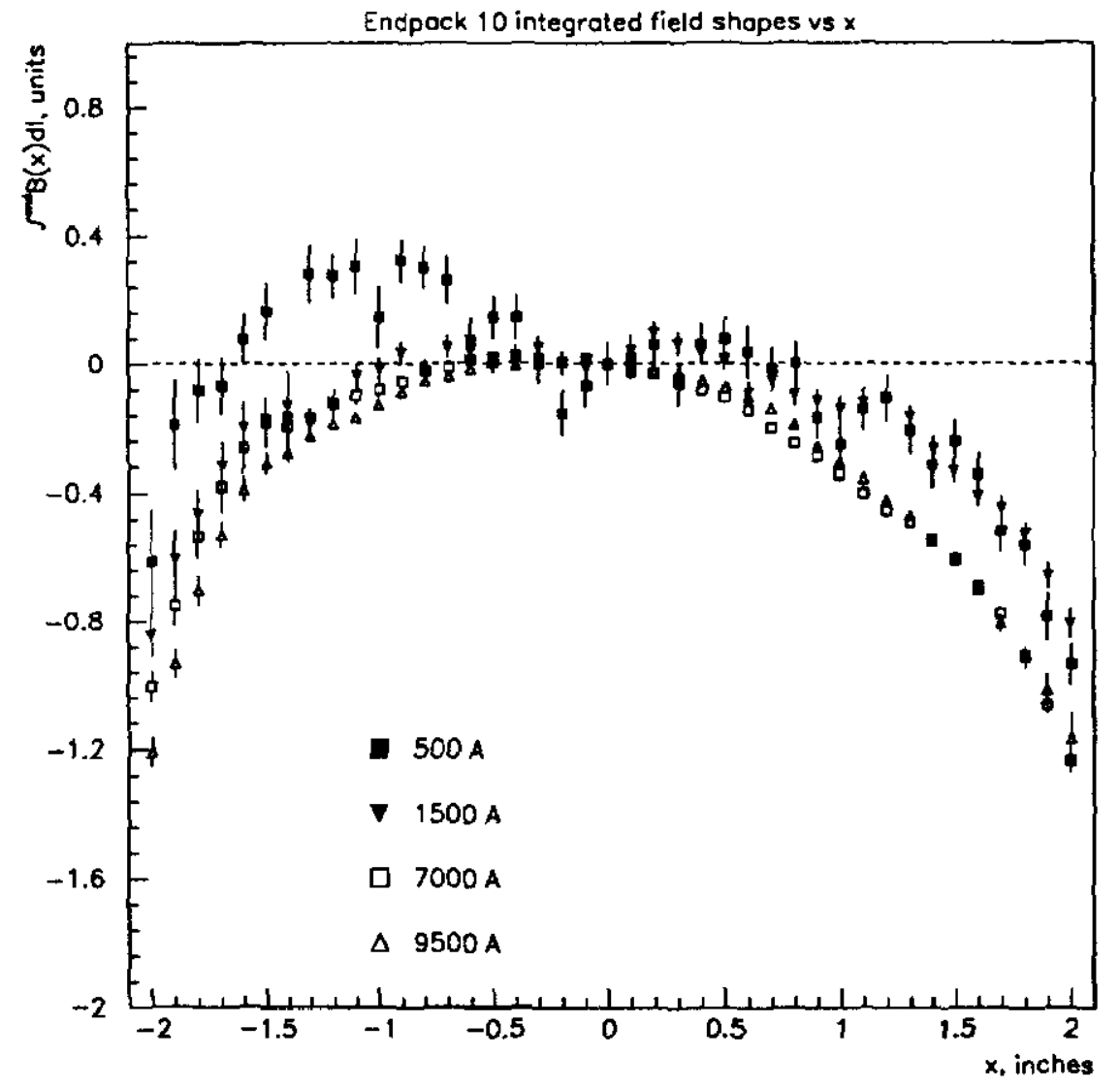

Figure 7: Integrated field shapes for Endpack 10. 


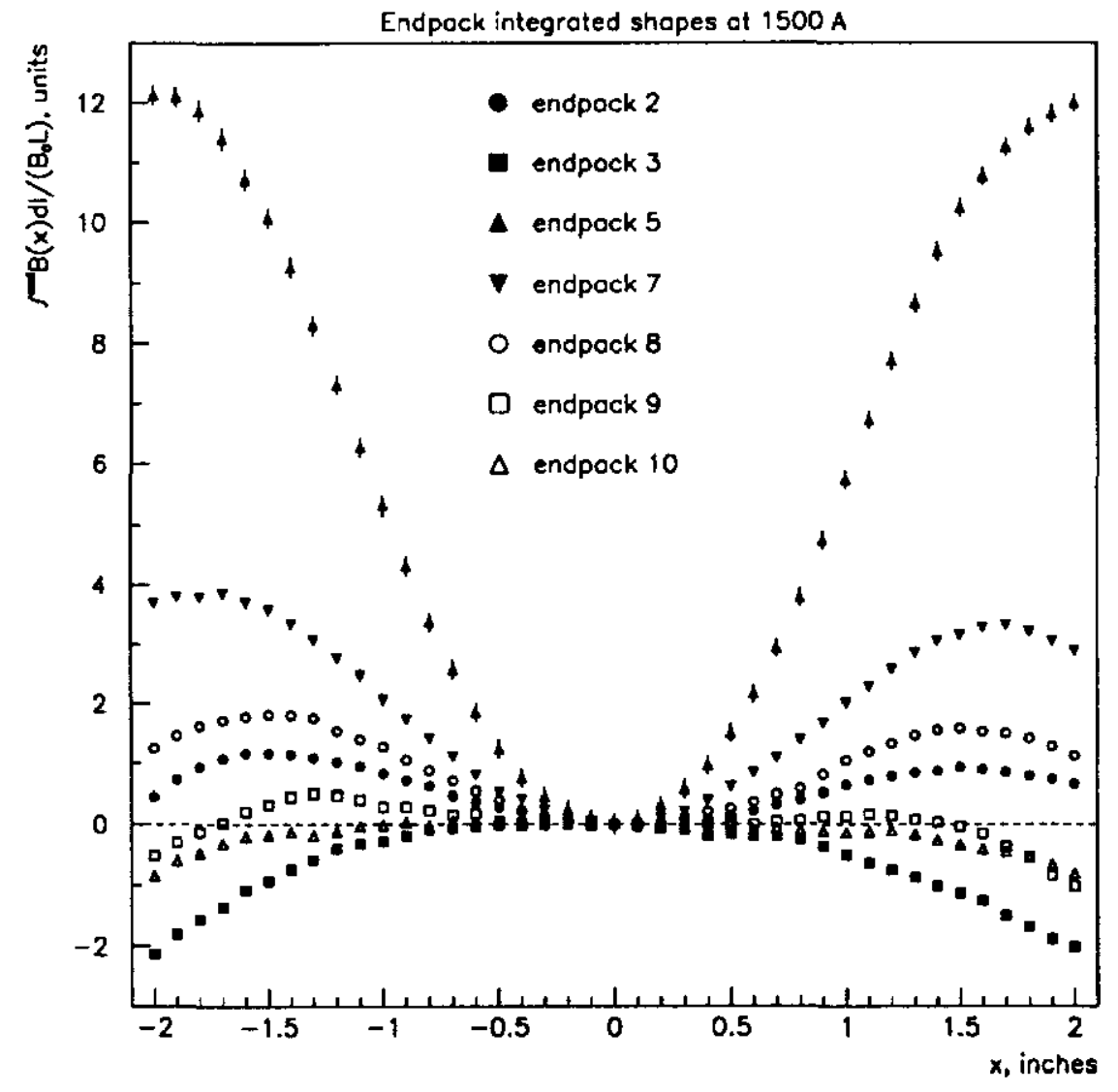

Figure 8: Integrated field shapes for all endpacks. 


\subsection{Estimation of normal harmonics}

We obtain the normal harmonics by fitting the function $\alpha(x)$, obtained from Eq. 3 to a polynomial:

$$
\alpha(x)=\sum_{n=0}^{p} J_{n} x^{n}
$$

Prior to performing the fit, the dipole term, $\alpha(0)$ is subtracted from $\alpha(x)$; this defines an endfield shape function, $s(x)=\alpha(x)-\alpha(0)$. We note that there is a simple relation between the effective length due to the endfield and this dipole term[1]:

$$
L_{\text {eff }}=\frac{\alpha(0)}{\beta(0)}
$$

In subtracting this constant from $\alpha(x)$, we also remove the systematic error due to the probe $z$ positioning error, since this source of error is the same for all values of $x$. The error in $s(x)$ may be approximated by:

$$
\sigma_{a}^{2}=\sigma_{\alpha(x)}^{2}+\sigma_{\alpha_{0}}^{2}-2 \rho \sigma_{\alpha(x)} \sigma_{\alpha_{0}}
$$

The correlation parameter, $\rho$, expresses the relative contribution of systematic effects to $\alpha(x)$. Because signal to noise increases with magnet current, we expect $\rho$ to increase with current. We found that if we estimate $\rho$ at current $i_{0}$, then at current $i_{1}$ the correlation parameter is:

$$
\rho_{1}=1-\frac{i_{0}}{i_{1}}\left(1-\rho_{0}\right)
$$

For several endpacks we estimated $\rho$ by comparing the errors in $\alpha(x)$ obtained by assuming a reasonable estimate for the $z$-positioning error, $\sigma_{z}$ [1], with the errors obtained assuming $\sigma_{z}=0$. This led to an estimate of $\rho=0.99$ at $500 \mathrm{~A}$ and $\rho=0.9995$ at $9500 \mathrm{~A}$.

The fit parameters $J_{n}$ in Eq. 6 are identified as the integrated normal harmonics over the end region, and are in units of [Tesla $\left.\cdot \mathrm{m} / \mathrm{inch}^{\mathrm{n}}\right]$. We chose to report results in terms of normalized harmonics, where the normalization is relative to the body dipole integrated over the length of the magnet:

$$
b_{n}=\frac{J_{n}}{B_{0} L}
$$

We used $\beta(0)$ for $B_{0}$ and 6.096 meters for $L$.

The value to choose for $p$ and the region in $x$ over which to perform the fit were chosen experimentally. Strictly speaking, one may not perform 


\begin{tabular}{|c|c|c|c|c|c|}
\hline current & $b_{1}$ & $b_{2}$ & $b_{3}$ & $b_{4}$ & $x_{v}^{2}$ \\
\hline 500 & $-.146 \pm .025$ & $+.578 \pm .034$ & $+.019 \pm .010$ & $-.197 \pm .010$ & 1.41 \\
1500 & $-.108 \pm .012$ & $+.428 \pm .016$ & $-.005 \pm .004$ & $-.157 \pm .004$ & 1.81 \\
7000 & $-.092 \pm .006$ & $+.402 \pm .008$ & $-.010 \pm .002$ & $-.168 \pm .002$ & 3.01 \\
9500 & $-.106 \pm .005$ & $+.305 \pm .007$ & $+.003 \pm .002$ & $-.149 \pm .002$ & 1.84 \\
\hline
\end{tabular}

Table 1: Harmonic coefficients for Endpack 9 using $p=4$

\begin{tabular}{|c|c|c|c|c|c|}
\hline current & $b_{1}$ & $b_{2}$ & $b_{2}$ & $b_{4}$ & $\chi_{2}^{2}$ \\
\hline 500 & $-.192 \pm .025$ & $+.142 \pm .034$ & $+.019 \pm .010$ & $-.081 \pm .010$ & 1.42 \\
1500 & $-.051 \pm .013$ & $-.046 \pm .017$ & $+.010 \pm .005$ & $-.038 \pm .005$ & 1.22 \\
7000 & $-.148 \pm .007$ & $-.151 \pm .009$ & $+.020 \pm .003$ & $-.025 \pm .003$ & 1.77 \\
9500 & $-.106 \pm .006$ & $-.160 \pm .008$ & $+.014 \pm .003$ & $-.029 \pm .003$ & 2.57 \\
\hline
\end{tabular}

Table 2: Harmonic coefficients for Endpack 10 using $p=4$

a harmonic fit over a region larger than a circle that just fits within the magnet aperture. Inside the body of the Main Injector magnet, the vertical aperture is $2.0^{\text {n }}$ and the horizontal aperture is wide open. Body field shapes are theoretically constrained to be fitted over regions $x_{\min }<x<x_{\max }$ such that $x_{\max }-x_{\min }<2.0^{\prime \prime}$. Note that we are only considering regions that are centered vertically $(y=0)$, and therefore skew harmonics can be neglected. At the ends, however, the vertical aperture opens up as a function of $z$, and the formal constraints on the fit region become less severe. We chose to fit over the region $|x|<2.0^{\prime \prime}$. With regard to the proper choice of $p$, we note that for dipoles one may expect important decapole contributions, which suggests choosing $p$ at least as large as 4; the next "allowed" harmonic after decapole is 14-pole, corresponding to $p=6$. We tried both 4 and 6 ; the fit to Endpack 10 indicates a need to use $p=6$ to achieve a good fit at the higher currents. Tables 1 and 2 list the harmonics obtained using $p=4$ for Endpacks 9 and 10. These values should be compared with the $p=6$ fits in Tables 8 and 9.

The fits were performed by the MINUIT program[2]; the fit parameters were calculated using the MIGRAD fitting option, and the error estimates were obtained by the MINOS option. In some instances, the fitting program complained that the error matrix was not positive definite. This can occur if the fit parameters are not all of the same order of magnitude and if the 
correlations are large. Both of these conditions were observed in our data. Correlations between the even harmonics $\left(b_{2}, b_{4}\right.$, and $\left.b_{6}\right)$ were observed to be 0.9 or higher.

\subsection{Results}

Tables 3 through 9 list the fits using $p=6$. At each current we list the normal harmonics $b_{1}$ through $b_{6}$. In these tables we use the convention that $b_{1}$ is the quadrupole coefficient, $b_{2}$ is the sextupole coefficient, etc. The reported values are in "units" at $1 \mathrm{inch}$, which is equivalent to $\left[10^{4} \times \mathrm{inch}^{-n}\right]$.

In figures 9 through 11 we display the results of the fits to the field shapes for Endpacks 2, 9, and 10. These figures give a qualitative indication that the fits are reasonably good.

Requirements based on tracking at injection (500 A magnet current) indicate a desire to have the endfield sextupole be $0.0 \pm .2$ units[4]. Only Endpack 10 appears to satisfy this requirement. 

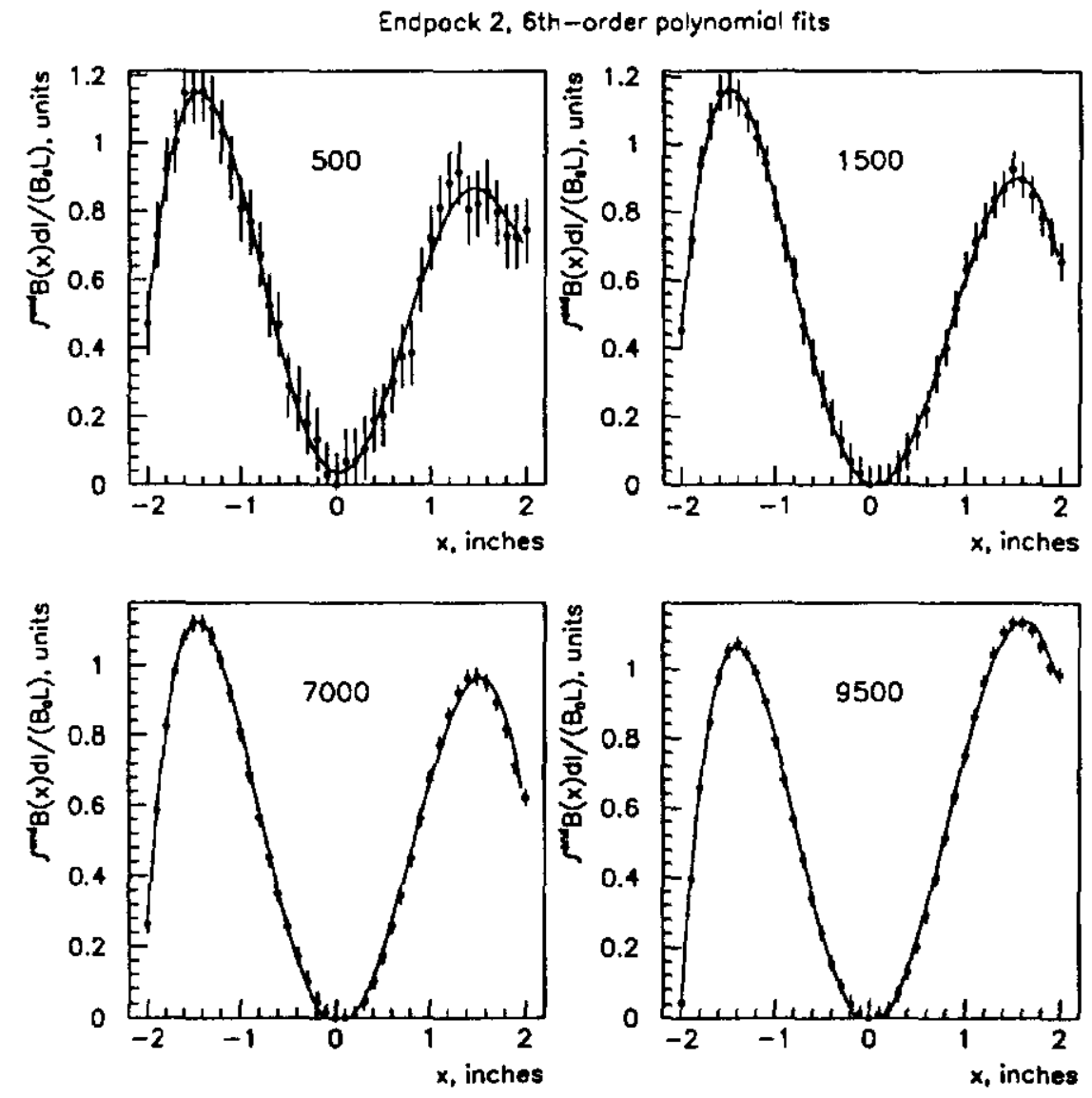

Figure 9: Results of fits to the Endpack 2 field shapes using $p=6$. A separate fit is done for each current. 
6th-order polynomial fits to Endpock 9
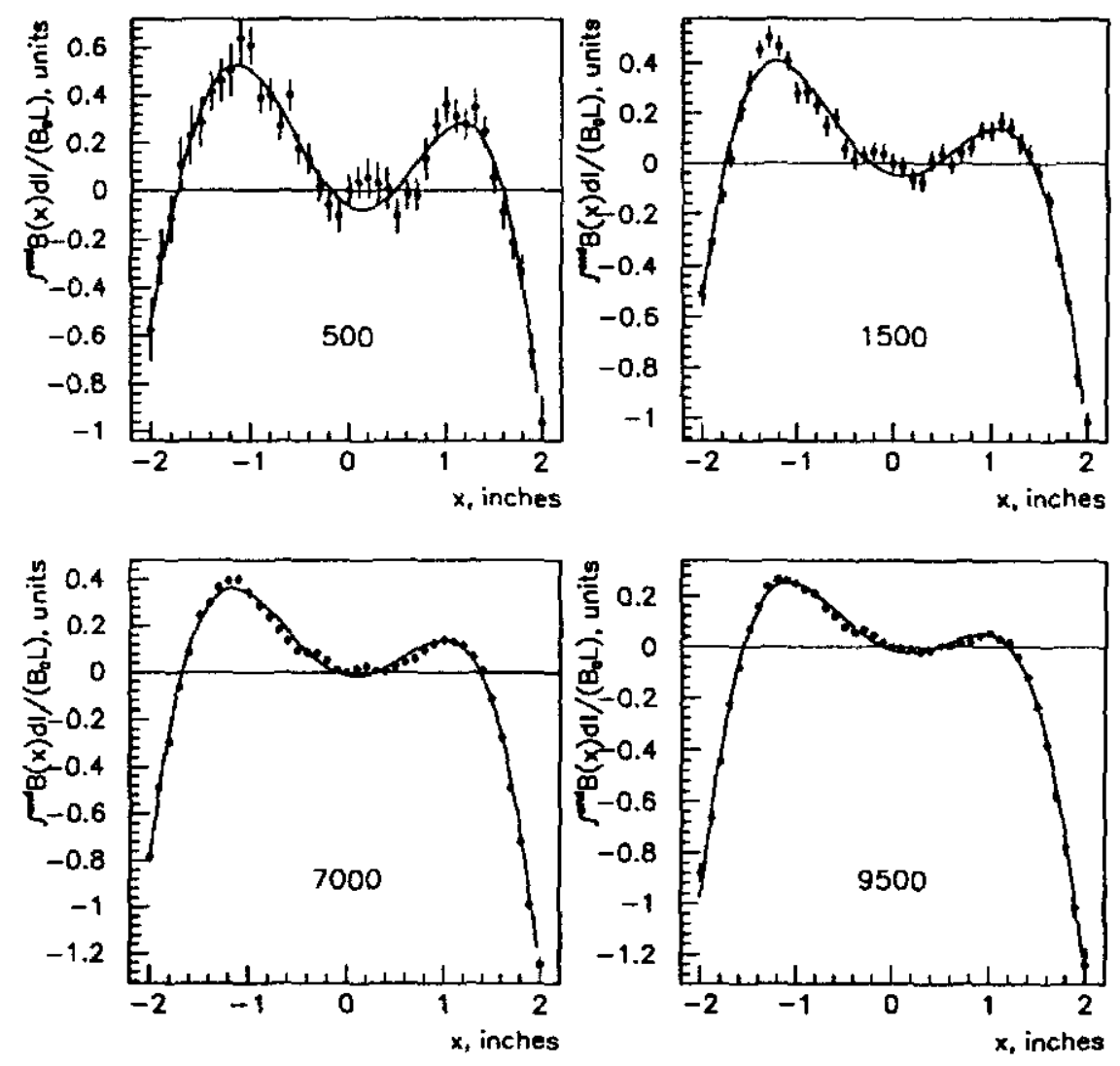

Figure 10: Fits to Endpack 9 field shapes $(p=6)$. 

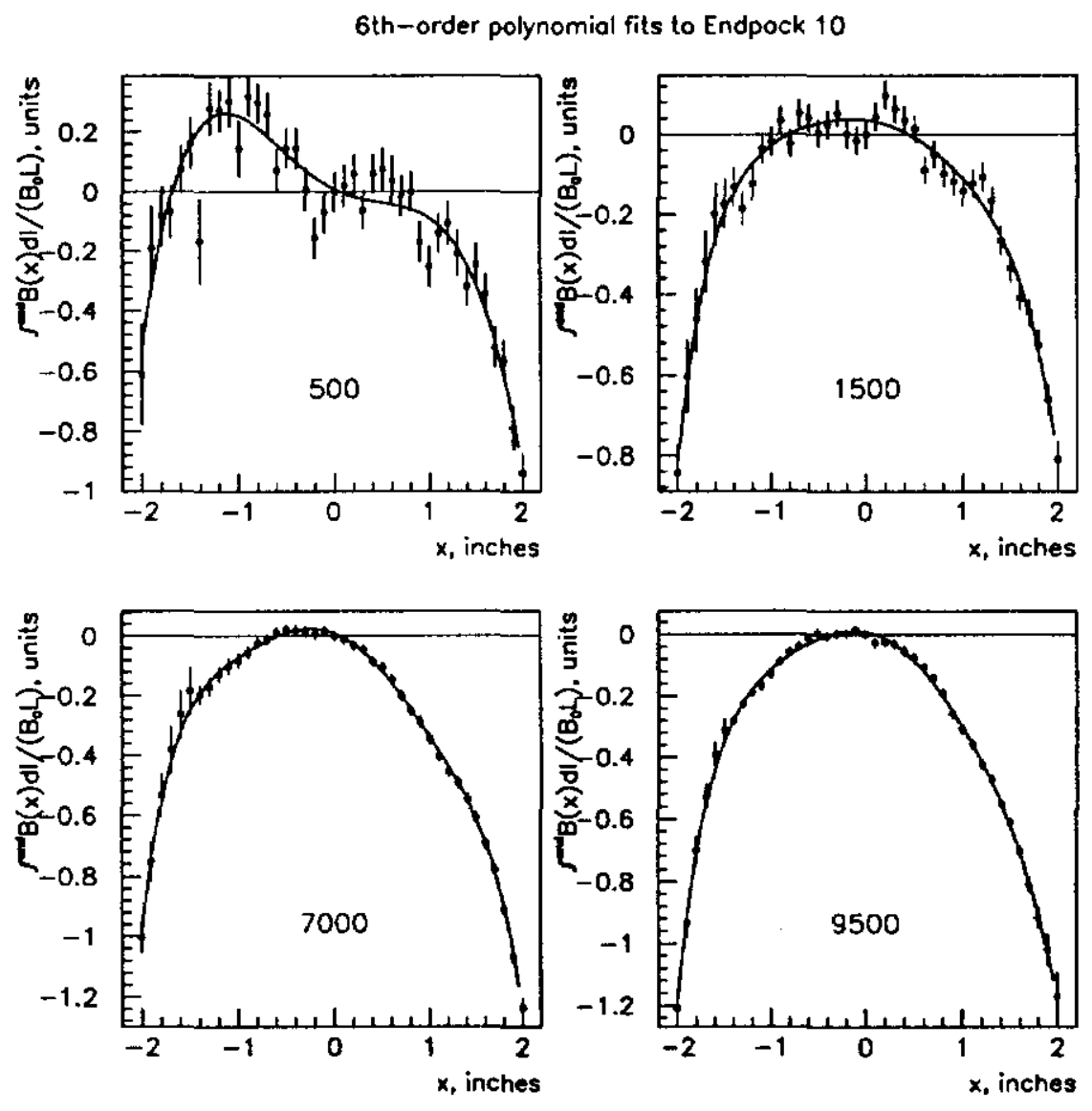

Figure 11: Fits to Endpack 10 field shapes $(p=6)$. 


\begin{tabular}{|c|c|c|c|c|c|c|c|}
\hline current & $b_{2}$ & $b_{2}$ & $b_{3}$ & $b_{4}$ & $b_{k}$ & $b_{6}$ & $x_{v}^{2}$ \\
\hline 500 & $-.052 \pm .054$ & $+1.041 \pm .092$ & $-.074 \pm .051$ & $-.331 \pm .060$ & $+.025 \pm .011$ & $+.027 \pm .010$ & 0.24 \\
1500 & $-.094 \pm .030$ & $+.048 \pm .052$ & $-.037 \pm .029$ & $-.239 \pm .034$ & $+.018 \pm .006$ & $+.009 \pm .006$ & 0.11 \\
7000 & $-.051 \pm .014$ & $+1.002 \pm .024$ & $-.043 \pm .013$ & $-.254 \pm .016$ & $+.019 \pm .003$ & $+.008 \pm .003$ & 0.41 \\
9500 & $-.018 \pm .012$ & $+1.062 \pm .020$ & $-.033 \pm .009$ & $-.282 \pm .013$ & $+.023 \pm .002$ & $+.012 \pm .002$ & 0.51 \\
\hline
\end{tabular}

Table 3: Normal harmonic coefficients for Endpack $2 ; b_{1}$ is the quadrupole component, $b_{2}$ is sextupole, etc.

\begin{tabular}{|c|c|c|c|c|c|c|c|}
\hline current & $b_{1}$ & $b_{2}$ & $b_{2}$ & $b_{4}$ & $b_{6}$ & $b_{6}$ & $x_{2}^{2}$ \\
\hline 500 & $-.064 \pm .059$ & $+.205 \pm .102$ & $-.007 \pm .058$ & $-.353 \pm .068$ & $-.001 \pm .013$ & $+.046 \pm .012$ & 1.39 \\
1500 & $-.129 \pm .030$ & $-.246 \pm .051$ & $-.000 \pm .028$ & $-.121 \pm .034$ & $+.010 \pm .006$ & $+.014 \pm .006$ & 0.42 \\
7000 & $-.100 \pm .014$ & $-.319 \pm .023$ & $-.014 \pm .013$ & $-.083 \pm .015$ & $+.011 \pm .003$ & $+.007 \pm .003$ & 0.74 \\
9500 & $-.063 \pm .012$ & $-.367 \pm .020$ & $+.007 \pm .011$ & $-.082 \pm .014$ & $+.009 \pm .002$ & $+.012 \pm .002$ & 0.54 \\
\hline
\end{tabular}

Table 4: Harmonic coefficients for Endpack 3

\begin{tabular}{|c|c|c|c|c|c|c|c|}
\hline current & $b_{1}$ & $b_{2}$ & $b_{3}$ & $b_{4}$ & $b_{3}$ & $b_{6}$ & $\chi_{2}^{2}$ \\
\hline 500 & $+.459 \pm .164$ & $+6.556 \pm .282$ & $-.285 \pm .157$ & $-.901 \pm .187$ & $+.036 \pm .033$ & $+.007 \pm .032$ & 0.15 \\
1500 & $+.365 \pm .088$ & $+6.455 \pm .152$ & $-.182 \pm .084$ & $-.849 \pm .101$ & $+.018 \pm .018$ & $-.003 \pm .017$ & 0.46 \\
7000 & $+.289 \pm .043$ & $+5.950 \pm .073$ & $-.158 \pm .041$ & $-.789 \pm .048$ & $+.016 \pm .009$ & $-.005 \pm .008$ & 1.44 \\
9500 & $+.258 \pm .036$ & $+5.166 \pm .061$ & $-.162 \pm .034$ & $-.695 \pm .041$ & $+.020 \pm .007$ & $-.003 \pm .007$ & 1.71 \\
\hline
\end{tabular}

Table 5: Harmonic coefficients for Endpack 5

\begin{tabular}{|c|c|c|c|c|c|c|c|}
\hline current & $b_{1}$ & $b_{2}$ & $b_{3}$ & $b_{4}$ & $b_{6}$ & $b_{6}$ & $\chi_{\nu}^{2}$ \\
\hline 500 & $+.058 \pm .063$ & $+2.730 \pm .113$ & $+.003 \pm .074$ & $-.650 \pm .084$ & $-.016 \pm .017$ & $+.046 \pm .016$ & 1.07 \\
1500 & $+.020 \pm .026$ & $+2.532 \pm .043$ & $-.070 \pm .027$ & $-.499 \pm .030$ & $+.004 \pm .006$ & $+.018 \pm .005$ & 0.46 \\
7000 & $+.058 \pm .012$ & $+2.382 \pm .021$ & $-.064 \pm .011$ & $-.489 \pm .014$ & $+.000 \pm .002$ & $+.019 \pm .002$ & 2.16 \\
9500 & $+.083 \pm .012$ & $+2.145 \pm .021$ & $-.098 \pm .012$ & $-.385 \pm .015$ & $+.006 \pm .003$ & $+.005 \pm .003$ & 1.76 \\
\hline
\end{tabular}

Table 6: Harmonic coefficients for Endpack 7 


\begin{tabular}{|c|c|c|c|c|c|c|c|}
\hline current & $b_{1}$ & $b_{2}$ & $b_{2}$ & $b_{1}$ & $b_{3}$ & $b_{6}$ & $x_{2}^{2}$ \\
\hline 500 & $-.150 \pm .039$ & $+1.413 \pm .067$ & $+.012 \pm .037$ & $-.301 \pm .044$ & $+.007 \pm .008$ & $+.004 \pm .008$ & 0.90 \\
1500 & $-.148 \pm .022$ & $+1.512 \pm .038$ & $+.030 \pm .021$ & $-.373 \pm .026$ & $-.001 \pm .004$ & $+.017 \pm .004$ & 1.21 \\
7000 & $-.149 \pm .012$ & $+1.322 \pm .020$ & $+.016 \pm .011$ & $-.322 \pm .014$ & $+.004 \pm .002$ & $+.010 \pm .002$ & 1.49 \\
9500 & $-.152 \pm .010$ & $+1.229 \pm .017$ & $+.030 \pm .010$ & $-.296 \pm .012$ & $+.001 \pm .003$ & $+.010 \pm .002$ & 1.20 \\
\hline
\end{tabular}

Table 7: Harmonic coefficients for Endpack 8

\begin{tabular}{|c|c|c|c|c|c|c|c|}
\hline current & $b_{1}$ & $b_{2}$ & $b_{2}$ & $b_{4}$ & $b_{3}$ & $b_{3}$ & $x_{v}$ \\
\hline 500 & $-.215 \pm .042$ & $+.750 \pm .074$ & $+.110 \pm .043$ & $-.334 \pm .051$ & $-.021 \pm .010$ & $+.026 \pm .009$ & 1.18 \\
1500 & $-.092 \pm .021$ & $+.488 \pm .036$ & $-.024 \pm .020$ & $-.200 \pm .023$ & $+.004 \pm .004$ & $+.008 \pm .004$ & 1.78 \\
7000 & $-.081 \pm .010$ & $+.448 \pm .017$ & $-.023 \pm .010$ & $-.202 \pm .011$ & $+.003 \pm .002$ & $+.006 \pm .002$ & 2.88 \\
9500 & $-.107 \pm .009$ & $+.325 \pm .015$ & $+.004 \pm .009$ & $-.166 \pm .011$ & $+.000 \pm .002$ & $+.003 \pm .002$ & 1.89 \\
\hline
\end{tabular}

Table 8: Harmonic coefficients for Endpack 9

\begin{tabular}{|c|c|c|c|c|c|c|c|}
\hline current & $b_{1}$ & $b_{2}$ & $b_{3}$ & $b_{4}$ & $b_{3}$ & $b_{6}$ & $x_{2}^{2}$ \\
\hline 500 & $-.159 \pm .042$ & $+.167 \pm .072$ & $-.021 \pm .043$ & $-.095 \pm .049$ & $+.009 \pm .010$ & $+.002 \pm .009$ & 1.47 \\
1500 & $-.033 \pm .021$ & $-.110 \pm .037$ & $-.014 \pm .023$ & $+.013 \pm .027$ & $+.006 \pm .005$ & $-.010 \pm .005$ & 1.16 \\
7000 & $-.126 \pm .011$ & $-.278 \pm .020$ & $-.017 \pm .013$ & $+.084 \pm .015$ & $+.008 \pm .003$ & $-.021 \pm .003$ & 0.32 \\
9500 & $-.056 \pm .010$ & $-.244 \pm .018$ & $-.053 \pm .011$ & $+.042 \pm .014$ & $+.017 \pm .003$ & $-.014 \pm .003$ & 0.69 \\
\hline
\end{tabular}

Table 9: Harmonic coefficients for Endpack 10 


\section{Effect of Endfields on Total Magnet Fieldshape}

The criteria for deciding the acceptability of an endpack must be based on assessing the contribution of the endfield to the total field quality of the magnet. In Figures 12 and 13 we show, for Endpacks 2 and 10, the relative shape of the body field, as measured by the 16' $4^{\prime \prime}$ Flatcoil[3]. The endfield shapes, normalized to $B_{0} L$, are superimposed. The figures also show an estimate of the total field shape, which is obtained by adding the body field shape to twice the endfield shape. We see that over the measured region $x<2.0^{\prime \prime}$, the total field shape when Endpack 10 is included experiences a maximum deviation of 1.2 units from the central value, while the situation for Endpack 2 is much less desirable (4.0 units maximum deviation). It is concluded that Endpack 10 produces an acceptable endfield for use with the Main Injector dipole. 


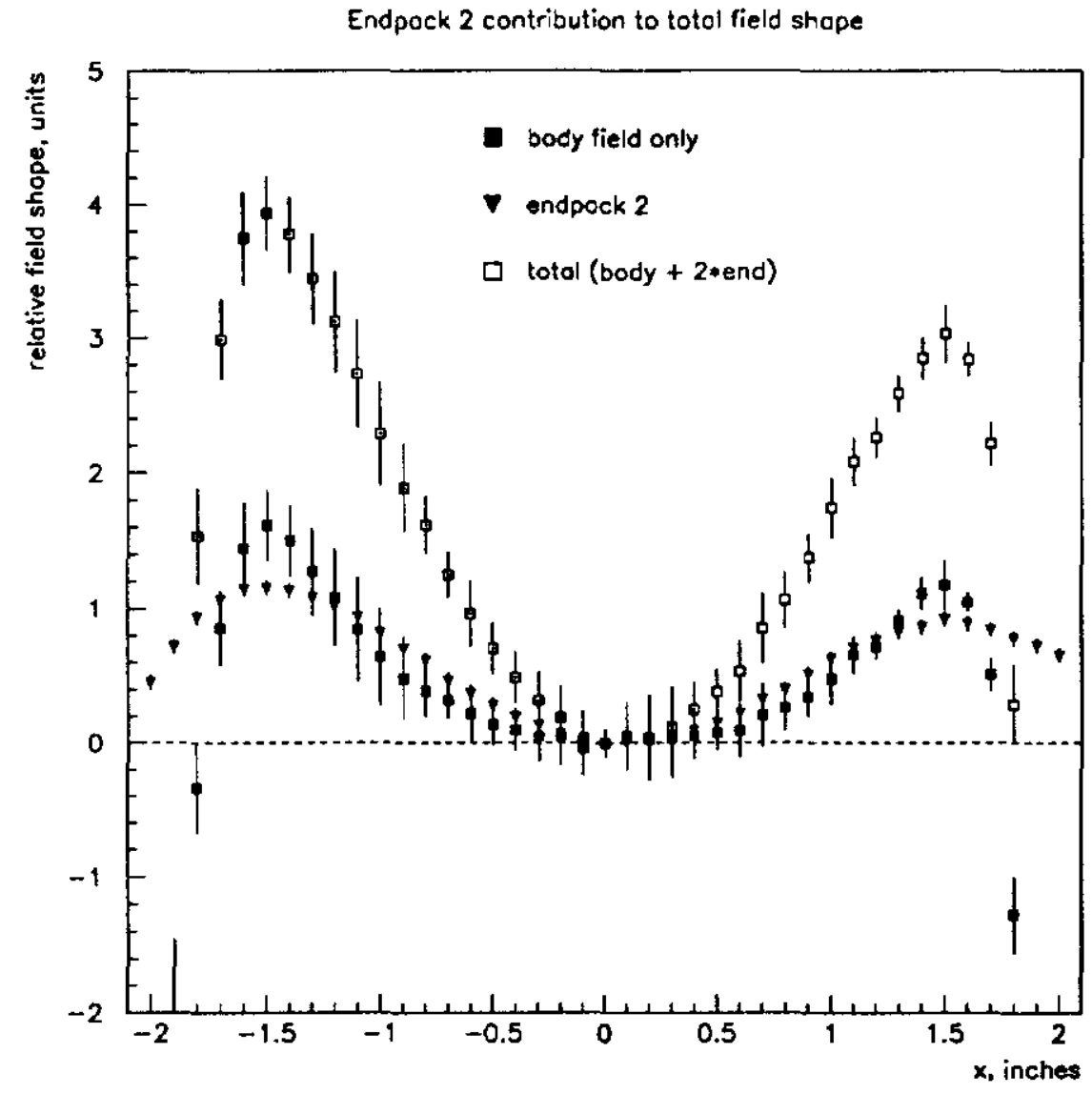

Figure 12: Measurement of the relative shapes of the body field and the integrated field of Endpack 2. The endfield shape has been normalized so that one obtains the total field shape by summing body field plus two ends. The endfield contributes significantly to the total field shape. 


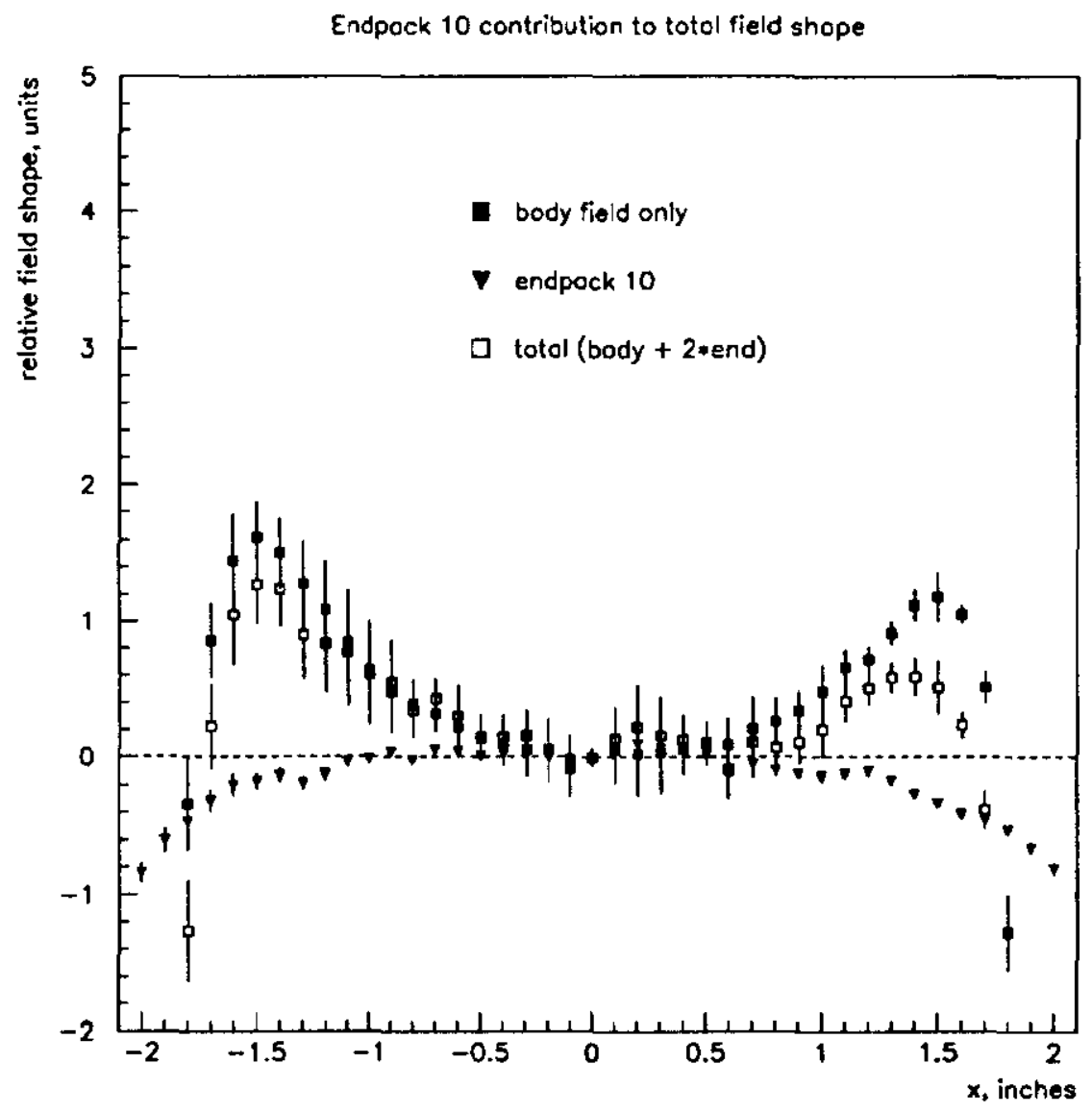

Figure 13: Measurement of the relative shapes of the body field and the integrated field of Endpack 10. This endpack has only a minor impact on the total field shape. 


\section{Acknowledgments}

The authors thank Dave Hartness, Shree Agrawal, and Mark Thompson for their excellent work in performing the measurement activities on these endpacks. We also thank Steve Helis for his efforts on construction of the probe. Additional assistance was provided by Butch Bianchi, Jim Garvey, Harold Stahl, and Peter Mazur.

\section{References}

[1] H. Glass et. al., "Effective Length Measurements of Main Injector Dipole Endpacks," MTF-92-018.

[2] MINUIT, Function Minimization and Error Analysis, PM0020.

[3] H. Glass et. al., "Body Field and Total Field Shape Measurements of the Main Injector Prototype magnets," MTF report (in preparation).

[4] S. Mishra and F. Harfoush, private communication. 